\title{
Tailoring the hydraulic impedance of out-of-plane micromachined electrospray sources with integrated electrodes
}

\author{
R. Krpoun, ${ }^{1, a)}$ K. L. Smith, ${ }^{2}$ J. P. W. Stark, ${ }^{2}$ and H. R. Shea ${ }^{1}$ \\ ${ }^{1}$ Ecole Polytechnique Fédérale de Lausanne, Microsystems for Space Technologies Laboratory, 2002 \\ Neuchâtel, Switzerland \\ ${ }^{2}$ School of Engineering and Materials Science, Queen Mary, University of London, Mile End Road, London \\ E1 4NS, United Kingdom
}

(Received 7 January 2009; accepted 14 March 2009; published online 21 April 2009)

\begin{abstract}
Hydraulic impedance is a critical parameter for the operation of electrospray emitters, and for preventing flooding when spraying from arrays of emitters. Controlling flow rate by tuning the flow impedance allows accessing different operating modes, such as droplet, ionic, or pulsating. We report on a method to tailor the hydraulic impedance of micromachined capillary out-of-plane emitters with integrated extractor electrodes by filling them with silica microspheres. Spraying the

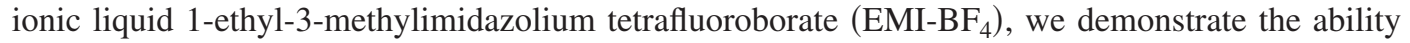
to tune from droplet emission to pure ion emission depending on microbead diameter, obtaining stable emission from single emitters and from arrays of 19 emitters. (C) 2009 American Institute of Physics. [DOI: 10.1063/1.3117191]
\end{abstract}

Large arrays of nanoelectrospray emitters capable of operating in pure ionic mode using ionic liquids are of great interest for space micropropulsion and as ion sources for future focused ion beam applications. The feasibility of large arrays of microfabricated silicon out-of-plane arrays of capillaries has been shown previously, ${ }^{1,2}$ but their operation has been difficult due to low hydraulic impedance, identified as a key parameter in the stability of electrosprays, ${ }^{3-5}$ and as a key parameter in determining ion to droplet ratio when spraying ionic liquids. Recent studies with the ionic liquids 1-ethyl-3-methylimidazolium tetrafluoroborate $\left(\mathrm{EMI}^{\left.-\mathrm{BF}_{4}\right)}\right.$ and 1-ethyl-3-methylimidazolium bis(trifluoromethylsulfonyl)imide (EMI-Tf $f_{2} \mathrm{~N}$, also referred to as EMI-Im), show that when sprayed from a capillary under vacuum conditions, a purely ionic regime (PIR) is only reached at low flow rates $(\ll 1 \mathrm{~nL} / \mathrm{s})$ while at higher flow rates mixed droplet-ion emission is observed. ${ }^{6-9}$

We have developed a method to tailor the hydraulic impedance in microfabricated silicon capillary emitters by creating a "porous" structure inside the capillary, thus combining the advantages of internally wetted capillaries with flow rate matching of externally wetted emitters. Similar to the method described by Valaskovic and Ehrenfeld ${ }^{10}$ for loading capillaries with diameters below $300 \mu \mathrm{m}$ with particulate materials, in our case without applying any pressure, the modification of the hydraulic impedance is achieved by introducing silica microspheres into the capillaries and fixing them by means of a silanization step using silicon tetrachloride $\left(\mathrm{SiCl}_{4}\right)$ gas. In addition, microfabricated extractor electrodes have been directly integrated onto the capillary arrays allowing for homogeneous spray conditions across the array. The capillaries have an inner diameter of $24 \mu \mathrm{m}$ and a height of $70 \mu \mathrm{m}$. Their fabrication has been described in detail in a companion paper. ${ }^{11}$

Retarding potential measurements with single integrated emitters spraying EMI-Tf ${ }_{2} \mathrm{~N}$, reported below, show operation in droplet mode without microspheres and ionic mode with

${ }^{\text {a)} E l e c t r o n i c ~ m a i l: ~ r e n a t o . k r p o u n @ a 3 . e p f l . c h . ~}$ them. Time-of-flight (TOF) spectra with arrays of 19 emitters with $5 \mu \mathrm{m}$ microspheres spraying EMI-BF ${ }_{4}$ demonstrate the operation of integrated out-of-plane capillary arrays in PIR.

Figure 1 shows an array of silicon capillary emitters, fabricated at the Ecole Polytechnique Fédérale de Lausanne, filled with $5 \mu \mathrm{m}$ microspheres. The microspheres dispersed in a water/2-propanol solution are introduced into the capillaries by placing a droplet of solution on the reservoir side. Capillarity pulls the beads into the capillary. Once filled, the solvent is dried and silanization performed. $\mathrm{SiCl}_{4}$ has a high volatility and reacts with water forming silicon dioxide $\left(\mathrm{SiO}_{2}\right)$ "bridges" between adjacent structures. Tests with $1 \mu \mathrm{m}$ spheres have shown similar filling results.

The tests with single capillaries were carried out in vacuum spraying EMI-Tf ${ }_{2} \mathrm{~N}$. The liquid was stored in a separate vacuum chamber and was pushed through a silica capillary to the emitter by applying a pressure. ${ }^{12}$ To avoid electrochemical degradation of the fuel the polarity of the spray was switched at a frequency of $0.25 \mathrm{~Hz}$ as suggested by Lozano and Martínez-Sánchez ${ }^{13}$ During our tests the emitters were biased to high voltage while the extractor electrodes were grounded. Current was measured using a Faraday cup. A suppression grid (biased at $-50 \mathrm{~V}$ ) was placed in front of the Faraday cup to avoid any "loss" of current in the form of secondary electrons.

The current-voltage characteristics of a single capillary emitter with $24 \mu \mathrm{m}$ inner diameter without and filled with

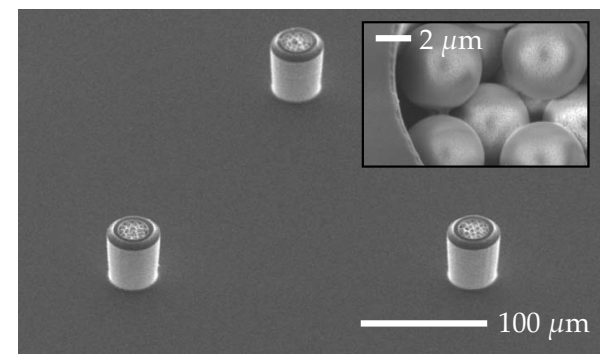

FIG. 1. SEM photographs of an array of silicon capillary emitters filled with $5 \mu \mathrm{m}$ diameter microspheres. The inset is a detail of the capillary tip. 


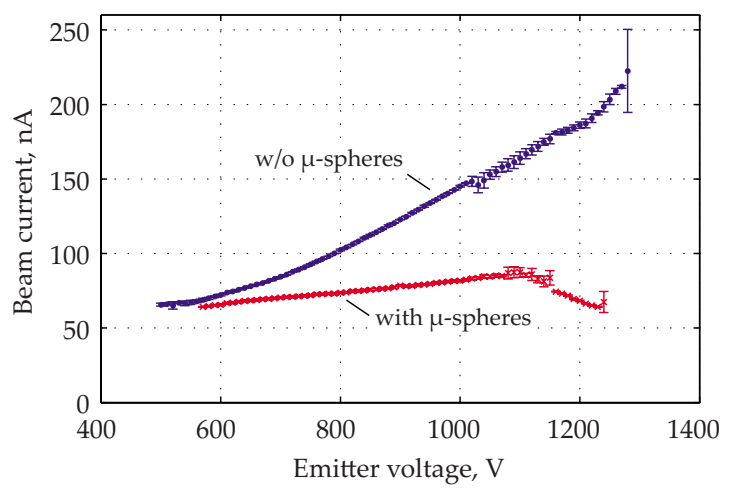

FIG. 2. (Color online) Current-voltage measurements of single capillary emitters without (top curve) and with silica microspheres (bottom curve). Error bars indicate the standard deviation of repeated measurements.

$5 \mu \mathrm{m}$ diameter microspheres is shown in Fig. 2. During the test backpressure was kept constant. The effect of the voltage on the current can be clearly seen for both filled and unfilled capillaries. Unlike a capillary without microspheres where the current increases constantly with voltage, for capillaries with microspheres, a decrease in current is observed above $\pm 1100 \mathrm{~V}$. This observation matches earlier measurements done with macroscopic capillaries spraying EMI-BF ${ }_{4}{ }^{14}$ One possible explanation for this behavior is that at higher electric pressure the operating mode transitions to a higher droplet fraction, which in general carries less current for a given backpressure.

The displayed error bars in Fig. 2 reflect the standard variation in the measured current during one test sequence, consisting in ramping up and down the voltage several times during approximately $10 \mathrm{~min}$. The error bars do not reflect the long term degradation of the source which has been discussed in Ref. 11. The test was stopped on the lower and upper end of the displayed voltage scale as instabilities set in.

The current-voltage curves were complemented by retarding potential measurements at different emitter voltages. The retarding voltage was applied to a grid placed between the suppression and a grounded grid. With the retarding grid in place particles are only collected for which $m v^{2} / 2>q V_{r}$, where $m$ is the particles mass, $v$ its velocity, $q$ its charge, and $V_{r}$ is the applied retarding voltage. The current versus retarding voltage measurements were interpolated and differentiated yielding the normalized density distribution plot shown in Fig. 3. The measured energy deficit for capillaries filled with beads is $120 \mathrm{eV}$. For capillaries without microspheres a

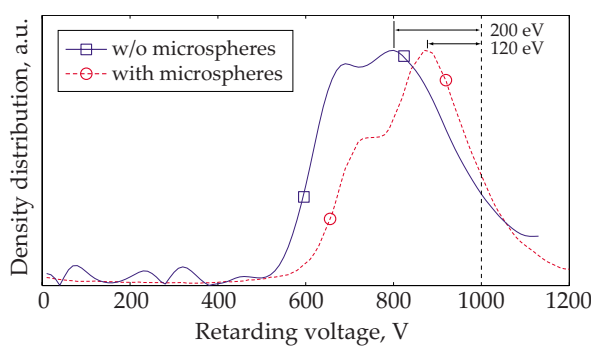

FIG. 3. (Color online) Energy density distribution for a capillary without and with microbeads spraying EMI-Tf ${ }_{2} \mathrm{~N}$, also referred to as EMI-Im. The smaller energy deficit and narrower energy distribution for the capillaries with microspheres indicate ionic operation, and the broader deficit mixed ion/droplet mode.

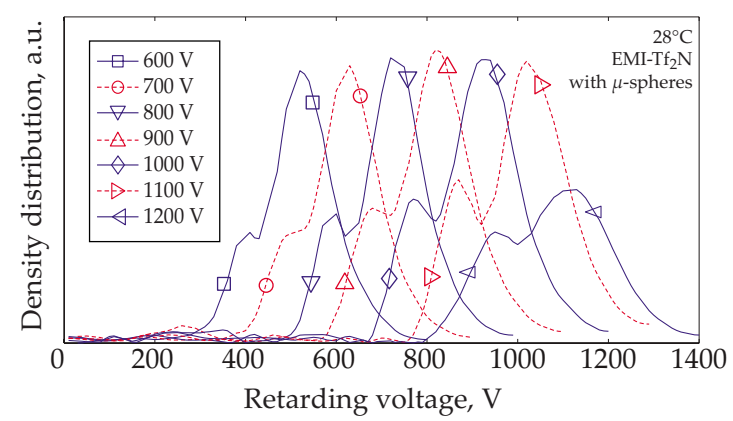

FIG. 4. (Color online) RPA measurements showing the density distributions for a capillary with microspheres for emitter voltages ranging from 600 to $1200 \mathrm{~V}$.

larger energy deficit $(200 \mathrm{eV})$ and broader distribution was observed. Analysis of the latter after spraying has revealed droplet depositions on the extractor electrode due to reflections from the retarding potential grid, which was not the case for the emitter filled with microspheres. The measured energy deficit for the capillary filled with beads is around $120 \mathrm{eV}$, but as Enloe and Shell ${ }^{15}$ pointed out, a grid does not represent an equipotential plane and the retarding voltage tends to "sag" between grid wires, thus limiting the resolution of the retarding potential analyzer, estimated to be around $5 \%-10 \%$ in our setup. Figure 4 shows additional retarding potential measurements done with a single capillary, filled with microspheres, at different emitter voltages. The shapes of the density distribution functions at voltages below $1100 \mathrm{~V}$ are practically identical and at $1200 \mathrm{~V}$ a slight broadening of the main peak can be observed, reflecting the change in behavior seen at $1100 \mathrm{~V}$ in Fig. 2.

Lozano $^{16}$ suggests that large energy deficits indicate the presence of a jet at the apex of the liquid cone. His explanation is that while a Taylor cone is an equipotential surface, equipotentiality cannot be guaranteed in the jet and a large drop in voltage should be expected across its total length, resulting in a higher energy deficit. This hypothesis has been recently confirmed by Gamero-Castaño 9 spraying from single capillary emitters. His retarding potential analysis results show an ionic peak with a large energy deficit and a 'bump' at energies above the ionic peak indicating the presence of droplets. On the other hand measurements with needle type emitters spraying EMI-Tf ${ }_{2} \mathrm{~N}$ in PIR have shown a single peak with an energy deficit of less than $10 \mathrm{eV}{ }^{16}$ In Fig. 3 we observe the same trend of a larger energy distribution for emitters without microspheres (operating in mixed regime) and of a lower energy deficit for capillaries with microspheres, possibly operating in a predominantly ionic regime.

To confirm operation in ionic regime, TOF measurements were carried out with arrays of 19 capillary emitters filled with microspheres. The dimensions of the capillaries were identical to the single emitter ones described in the previous paragraphs. The array pitch was $250 \mu \mathrm{m}$. The beam was interrupted by a potential barrier which was controlled using a high-speed switch pulse generator (DEI PVX4110) with a rise/fall time below $60 \mathrm{~ns}$. The drift length of the particles between the barrier and the cup was $L_{\mathrm{TOF}}$ $=410 \mathrm{~mm}$. The charge to mass ratio of a particle can be computed from its velocity and beam potential. Figure 5 shows the current versus atomic mass of an array of emitters spraying the ionic liquid $\mathrm{EMI}-\mathrm{BF}_{4}$. TOF traces were taken 


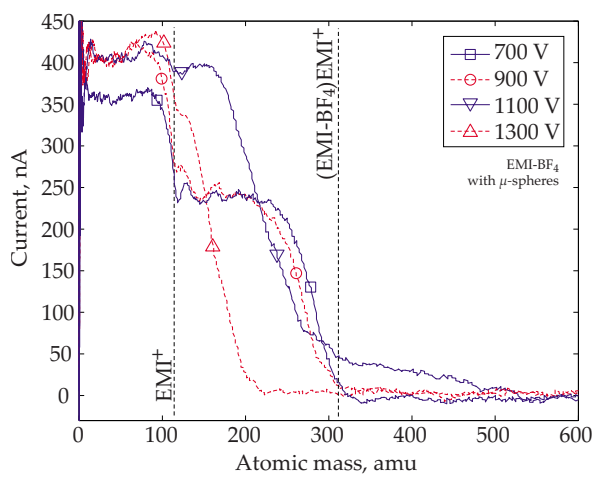

FIG. 5. (Color online) TOF traces recorded with a 19 emitter array with capillaries having a $24 \mu \mathrm{m}$ inner diameter filled with $5 \mu \mathrm{m}$ microspheres with an extractor electrode with $200 \mu \mathrm{m}$ holes space at $90 \mu \mathrm{m}$ from the capillary tip. Current trace as a function of atomic mass assuming the beam voltage to be equal to the emitter voltage. The masses for the $\mathrm{EMI}^{+}$monomer and the $\left(\mathrm{EMI}^{-} \mathrm{BF}_{4}\right) \mathrm{EMI}^{+}$dimer are marked as dotted lines.

for different emitter voltages. The corresponding atomic mass was calculated from the TOF measurements assuming simple ionization of the particles and a beam potential equal to the emitter voltage. The two dashed lines indicate the atomic mass of the monomer $\mathrm{EMI}^{+}(111 \mathrm{amu})$ and the dimer

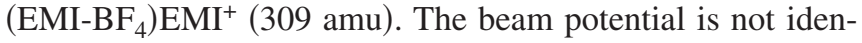
tical to the emitter voltage, due to energy losses from the formation of the cusp or jet especially for the $1300 \mathrm{~V}$ curve. This explains why beam current decreases before the corresponding mass is reached.

The results show a beam composed primarily of monomers and dimers, and show the absence of droplets. It might be argued that a great part of the beam is lost to the walls as the emission cone half-angle is around $30^{\circ}$ and that droplets might therefore not be seen under these circumstances. Prior work done with capillary emitters without microspheres doing on-axis TOF measurements ${ }^{6,7,9}$ shows that when droplets are present they can be measured on the beam axis. These results give us good confidence that no droplets are present in the beam.
The presented results prove the functionality of integrated out-of-plane electrospray arrays using silica microspheres to increase hydraulic impedance thus matching the emitted and supplied liquid flow. The obtained energy distribution and TOF spectra show a thruster operating in ionic mode. The specific impulse has been computed from the TOF traces and is greater than $3500 \mathrm{~s}$ for extractor voltages of $1200 \mathrm{~V}$ which corresponds to particle speeds of $35 \mathrm{~km} / \mathrm{s}$. Future work will focus on fabricating large arrays with thousands of emitters allowing to obtain a large thrust and on lifetime testing.

The authors would like to thank the European Space Agency for partially funding this work.

${ }^{1}$ M. Alexander, J. P. W. Stark, K. L. Smith, B. Stevens, and B. Kent, J. Propul. Power 22, 620 (2006).

${ }^{2}$ R. Krpoun, M. Räber, and H. R. Shea, Proceedings on the 21st IEEE International Conference on Micro Electro Mechanical Systems, Tucson, 2008 (unpublished), Vol. 0234, pp. 964-967.

${ }^{3}$ Y. X. Wang, J. W. Cooper, C. S. Lee, and D. L. De Voe, Lab Chip 4, 363 (2004)

${ }^{4}$ W. Deng, J. Klemic, X. Li, M. Reed, and A. Gomez, J. Aerosol Sci. 37, 696 (2006).

${ }^{5}$ L. F. Velásquez-García, A. I. Akinwande, and M. Martínez-Sánchez, J. Microelectromech. Syst. 15, 1260 (2006).

${ }^{6}$ I. Romero-Sanz, R. Bocanegra, and J. Fernandez de la Mora, J. Appl. Phys. 94, 3599 (2003).

${ }^{7}$ I. Guerreo, R. Bocanegra, F. Higuera, and J. Fernández De La Mora, J. Fluid Mech. 591, 437 (2007).

${ }^{8}$ D. Garoz, C. Bueno, C. Larriba, S. Castro, I. Romero-Sanz, J. De La Mora, Y. Yoshida, and G. Saito, J. Appl. Phys. 102, 064913 (2007).

${ }^{9}$ M. Gamero-Castaño, Phys. Fluids 20, 032103 (2008).

${ }^{10}$ G. Valaskovic and E. E. Ehrenfeld, U.S. Patent No. 6,395,183 (28 May 2002).

${ }^{11}$ R. Krpoun and H. R. Shea, J. Micromech. Microeng. 19, 045019 (2009).

${ }^{12} \mathrm{R}$. Krpoun, Ph.D. thesis, Ecole Polytechnique Fédérale de Lausanne, 2008.

${ }^{13}$ P. Lozano and M. Martínez-Sánchez, J. Colloid Interface Sci. 280, 149 (2004).

${ }^{14}$ J. Jhuree, M. S. Alexander, and J. P. W. Stark, Proceedings of the 30th International Electric Propulsion Conference, Florence, Italy, 2007 (unpublished).

${ }^{15}$ C. L. Enloe and J. R. Shell, Rev. Sci. Instrum. 63, 1788 (1992).

${ }^{16}$ P. Lozano, J. Phys. D 39, 126 (2006). 